\title{
La littératie scolaire : exigences et malentendus. Les registres de travail des élèves
}

School literacy: Demands and misunderstandings. Pupils'work registers

\section{Élisabeth Bautier et Patrick Rayou}

\section{(2) OpenEdition}

\section{Journals}

Édition électronique

URL : http://journals.openedition.org/educationdidactique/1721

DOI : 10.4000/educationdidactique.1721

ISSN : 2111-4838

Éditeur

Presses universitaires de Rennes

\section{Édition imprimée}

Date de publication : 30 octobre 2013

Pagination : 29-46

ISBN : 978-2-7535-3255-7

ISSN : 1956-3485

Référence électronique

Élisabeth Bautier et Patrick Rayou, «La littératie scolaire : exigences et malentendus. Les registres de travail des élèves », Éducation et didactique [En ligne], 7-2 | 2013, mis en ligne le 31 octobre 2015,

consulté le 19 avril 2019. URL : http://journals.openedition.org/educationdidactique/1721 ; DOI :

10.4000/educationdidactique. 1721 


\title{
LA LITTÉRATIE SCOLAIRE : EXIGENCES ET MALENTENDUS LES REGISTRES DE TRAVAIL DES ÉLÈVES
}

\author{
Élisabeth Bautier, laboratoire Circeft-EScol, université Paris 8 \\ Patrick Rayou, laboratoire Circeft-EScol, université Paris 8
}

Cet article propose un retour sur nos analyses antérieures relatives à la façon dont les élèves peuvent entrer dans des malentendus à l'occasion d'activités qui leur sont proposées en matière de compréhension de l'écrit. Ces analyses semblent devoir être affinées pour rendre compte des difficultés qu'ils éprouvent lors même qu'ils mobilisent des savoirs et des méthodes qui leur ont été enseignés. Nous introduisons pour cela la notion de " registres » (cognitif, culturel, symbolique) que les élèves doivent, au-delà du registre strictement scolaire, convoquer à bon escient. Nous la mettons au travail sur les réponses des élèves à deux questions de l'enquête PISA, emblématiques selon nous, des tâches aujourd'hui proposées aux élèves sur des textes composites et non linéaires. Nous proposons des pistes d'analyses en termes de configuration de registres, qu'il s'agisse de la manière dont l'institution scolaire organise les savoirs qu'elle dispense ou de celle dont les élèves s'en saisissent de manière plus ou moins heureuse pour eux.

Mots-clés: curriculum, inégalités, littératie, malentendus, registres.

\section{School literacy: Demands and misunderstandings. Pupils'work registers}

This article proposes a re-analysis of our previous work on students' misunderstandings during reading activities. Our previous conclusions must be refined to account for their difficulties even when they mobilize knowledge and methods they have been taught. For that purpose, we introduce the concept of registers (cognitive, cultural, symbolic), to which, beyond the academic register, students need to resort wisely in order to suceed. We examine the fecondity of this notion through the study of answers to two questions of the PISA questionnaire which are for us emblematic of the tasks proposed to students on composite and non-linear texts. We offer new avenues worth of exploring in terms of the configuration of registers, taking into account the ways in which school organises knowledge and the extent to which students grasp them.

Keywords: curriculum, inequality, literacy, misunderstandings, registers. 


\section{INTRODUCTION}

Depuis une dizaine d'années les enquêtes de PISA (évaluation internationale de la compréhension de l'écrit, des mathématiques et des sciences) montrent chez les élèves français ${ }^{1}$, en compréhension de l'écrit et en culture mathématique, une baisse constante des performances moyennes, au sein de laquelle la situation de ceux qui ne possèdent pas les compétences minimales ne cesse de s'aggraver ${ }^{2}$. Ce sont précisément ces difficultés éprouvées dans les situations scolaires et progressivement transformées en inégalités sociales à l'école, que notre équipe de recherche $\mathrm{EScol}^{3}$, puis le réseau RESEIDA ${ }^{4}$ qu'elle a fondé, se sont attachés à comprendre, et à élucider ${ }^{5}$. Nous avons avancé la notion de malentendu (Bautier \& Rochex, 2007, Bautier \& Rayou, 2013) pour décrire les différences le plus souvent qualitatives entre les élèves qui « réussissent » et ceux dont les difficultés s'accumulent au long de la scolarité. Cependant, compte tenu des évolutions curriculaires (Bautier, 2010) et des dispositifs et supports de travail illustrées de façon emblématique par les objectifs et les formes de l'évaluation internationale PISA, il nous semble nécessaire de revenir de façon plus précise et plus opératoire, plus adaptée aussi aux nouvelles difficultés rencontrées par les élèves, sur ce que demande aujourd'hui l'école et sur la façon dont ils répondent à ces demandes. Nos premières investigations dans ce domaine permettent de faire l'hypothèse d'une inégalité sociale des élèves devant ces évolutions curriculaires. Comprendre les logiques qui sous-tendent les réponses différentes des élèves relevant de socialisations différenciées nous semble une nécessité si on veut espérer des réponses adaptées des enseignants à ces difficultés.

Ainsi, dans une première période de recherches à différents niveaux scolaires (dont une première recherche effectuée à partir du corpus constitué par l'évaluation PISA de la compréhension de l'écrit $\left.{ }^{6}\right)$, nos travaux avaient mis en évidence que les élèves, face à des documents et consignes de travail, mobilisaient leurs expériences, leurs valeurs morales, leurs connaissances et pratiques ordinaires. Nous montrions également que ces mobilisations pouvaient faire obstacle à la compréhension des textes et plus encore à la possibilité de répondre «selon les attendus scolaires » aux consignes. Nous avions qualifié de "malentendus", le fait que, de leur côté, ces élèves estimaient souvent à tort faire ce qu'il fallait et que, de leur côté, les enseignants pensaient indûment que la sollicitation des opinions et expériences des élèves leur facilitait la tâche scolaire lorsqu'ils étaient en difficulté.

Dans la perspective de mieux identifier les phénomènes à l'œuvre au sein même du travail de l'élève, nous avons ensuite catégorisé différents types de savoirs et connaissances que les situations et documents devaient conduire les élèves à mobiliser et la nature des différents enjeux de cette mobilisation. Nous avions pu faire l'hypothèse que ces connaissances, savoirs et enjeux mobilisés permettaient de différencier les élèves. Celle-ci s'est révélée exacte pour une partie des élèves, ce qui nous conduit aujourd'hui à affiner la nature des malentendus en cause lorsque des élèves ne semblent pas construire de savoirs dans les situations que les enseignants et les manuels proposent au demeurant avec cette finalité. C'est cette caractéristique qui nous fait aujourd'hui déplacer le raisonnement et penser les attentes de l'école, comme le travail des élèves, en termes de pluralité de registres (culturel, cognitif, symbolique) à convoquer au-delà du registre spécifiquement scolaire. Car la nécessité, pour les élèves, de les hiérarchiser et tisser à « bon escient $»$ est une cause majeure de différences entre eux. Certains y parviennent, quand d'autres soit ne savent pas pluraliser les registres qui sont hétérogènes tant sur le plan cognitif que sur celui des savoirs et connaissances convoqués, ou sur celui plus symbolique de la construction de soi comme sujet, soit ne savent pas les identifier et les hiérarchiser en fonction des situations proposées.

En effet, notre analyse des documents, consignes, situations de travail montre que les attentes scolaires, de plus en plus implicites, offrent aujourd'hui un paysage apparemment paradoxal. Là où l'École française s'était construite dans la clôture symbolique et les savoirs strictement disciplinaires avec leur accompagnement d'exercices et de pratiques d'exercisation, spécifiques de chaque discipline, l'École très contemporaine fait éclater ces références par une porosité de plus en plus grande non seulement entre les disciplines scolaires, mais aussi entre l'École et ce qui n'est pas elle. Ainsi, la régionalisation des savoirs (Bernstein, 2007, Bautier, 2010, Stavrou, 2008) conduit l'école à traiter des questions sociales (l'environnement, la santé, par exemple) sans tradition disciplinaire. De même, le développement lent mais sûr depuis le Romantisme d'une culture de l'expression ${ }^{7}$ qui entre en cohérence avec les théo- 
Élisabeth Bautier et Patrick Rayou

ries constructivistes et non plus transmissives de l'apprentissage, sollicite les élèves sur le registre de la subjectivité et transforme les représentations ordinaires qu'ils se font de ce qu'est un texte qui énonce des savoirs, de sa légitimité et de la place énonciative de leur auteur. Et plus encore, la nécessité pour l'école de permettre à tous de « traiter » la pluralité des documents numériques et papier dans une forme devenue aujourd'hui délinéarisée et plurisémiotisée (ce qu'évalue PISA) a conduit enseignants, auteurs de manuels, d'albums de littérature de jeunesse à utiliser et produire des supports de travail qui sont euxmêmes extrêmement complexes. Les caractéristiques dominantes de ces textes résident dans leur hétérogénéité sémiotique (photographies, schémas, textes, $\mathrm{BD}$, graphies plurielles...) d'une part, d'autre part, dans leur contenu pluriel faisant appel à des savoirs quotidiens, autant qu'académiques, à de la lecture autant qu'à des sollicitations d'écritures hétérogènes, écriture-repérage et recopiage, écriture d'élaboration et de tissage, et même de rapiéçage (voir ci-après). Ils convoquent en effet des systèmes sémiotiques variés, des contenus divers et pas toujours des contenus de savoirs, brouillent les pistes de compréhension (voir ci-après et Bautier et alii, 2012). De plus, ils n'offrent guère aux élèves des exemples de textes à produire, de place énonciative à occuper de façon claire (qui parle et comment doit-il parler pour dire quoi ? L'élève ou le jeune?).

Ce contexte oblige à ne plus pouvoir analyser les productions des élèves comme se situant ou non dans LA bonne interprétation cognitive et culturelle du travail à effectuer. Il est nécessaire d'analyser ces productions ainsi que les supports qui les suscitent à la lumière du jeu cognitif et langagier attendu. Car la porosité qu'ils supposent entre les savoirs scolaires et les connaissances expérientielles et médiatiques, entre les sollicitations sur le registre de l'intériorité et le formalisme plus scolaire des genres discursifs élaboratifs n'est pas maîtrisée par tous les élèves. Le brouillage de valeur entre ces différents genres est grand pour les élèves quand le discours de chacun vaut aujourd'hui le discours du groupe et du collectif (Chiss, 2011).

Avant de montrer et d'étudier la façon dont des élèves se comportent face aux épreuves de PISA, nous proposons ci-dessous une analyse de ce que sont pour nous les malentendus, en particulier à partir des registres des apprentissages, ainsi qu'une approche de ce que sont aujourd'hui les textes utilisés à l'école.

\section{DES MALENTENDUS COMPLEXES}

\section{Malentendus absolus et malentendus relatifs}

Nous estimons qu'une des sources fondamentales de différenciation dans les apprentissages sont les malentendus par lesquels enseignants et élèves mélangent inopportunément les logiques cognitives spécifiques de l'école et les logiques sociales (Bautier \& Rochex, 2007 ; Bautier \& Rayou, 2012). Ces malentendus sont d'autant plus prégnants que la tradition républicaine de l'école considère que la clôture sur elle-même d'une école déterritorialisée est la garantie de ce que les influences sociales restent à sa porte. De fait, la signification même donnée aux apprentissages qui conduisent à la réussite scolaire dépend en grande partie des origines sociales des élèves. Comme l'ont révélé les enquêtes sur leur rapport au savoir, les élèves qui attendent le plus de l'école tendent à ne pas voir qu'elle propose des savoirs dont le bénéfice des apprentissages ne se perçoit pas tout de suite (Charlot, Bautier \& Rochex, 1992 ; Charlot, 1997). N'étant pas faite pour construire l'employabilité des élèves à laquelle elle contribue néanmoins, l'école peut être un lieu de malentendus en suscitant des formes d'instrumentalisme à son égard. La focalisation sur ses objectifs d'intégration professionnelle et sociale peut ainsi faire perdre de vue qu'il y a des manières proprement scolaires d'apprendre et que s'acquitter simplement des tâches prescrites peut paradoxalement faire passer à côté du sens même d'activités supposées former les élèves sur le long terme (Bonnéry, 2007).

De telles analyses pourraient cependant laisser penser que les élèves en réussite adhèrent pleinement aux objectifs et modes d'apprentissage de l'école et ne voient les possibles bénéfices sociaux qu'elle procure que comme des conséquences secondaires et lointaines tandis que les autres y mènent une existence au jour le jour dans l'inconscience des véritables enjeux. Or, nombre d'élèves socialement et culturellement bien dotés peuvent réussir scolairement tout en n'adhérant que superficiellement aux idéaux de connaissance de l'école. Conscients, pour une partie d'entre eux, que celle-ci n'est qu'une ressource, certes importante, parmi celles que leur entourage met à leur disposition, ils se trouvent aussi, selon le degré de mixité sociale des établissements qu'ils fréquentent, pris dans une expérience adolescente et des modes de socialisation horizontale qui les 
conduisent à tempérer les attentes scolaires (Barrère, 2011). L'instrumentalisme est donc une attitude relativement partagée qui cultive des malentendus sur les mobilisations réellement attendues. Il n'a cependant pas la même signification pour les élèves pour qui l'école est le seul recours et pour ceux qui ont compris la nécessité de se maintenir dans le système scolaire et de souscrire dans la durée à ses demandes, même si c'est a minima.

Ces considérations contraignent à distinguer des malentendus qu'on pourra, comme dans le premier cas, appeler absolus d'autres, comme dans le second, qu'on considèrera comme relatifs. Pour mieux comprendre ce qui les différencie et la façon dont ils se construisent, nous supposons d'une part que les logiques sociales et celles de l'apprentissage sont plus complexes et plus métissées que nous ne le disions jusqu'à présent. L'école n'est pas en effet, et de moins en moins, un empire cognitif dans un empire social, même si elle construit et évalue toujours des manières de faire spécifiques. D'autre part, les rapports entre ses diverses demandes évoluent au cours du curriculum. Les rythmes différents de montée vers des savoirs de plus en plus génériques diffèrent selon les systèmes éducatifs (Bernstein, 1975) et le comportement adéquat consiste à sentir, sinon identifier, les moments où se situent les modifications majeures d'exigences.

\section{Des registres de l'apprentissage}

Nous proposons de considérer que les apprentissages scolaires mettent en jeu des registres différents et complémentaires de mobilisation des élèves ${ }^{8}$. Le registre proprement scolaire concerne les savoirs en jeu dans la classe, qui relèvent, au moins jusqu'à la fin de l'enseignement secondaire, d'une didactisation. Ce registre est lui-même l'intersection de trois autres registres qui l'excèdent. Le premier est cognitif en ce qu'il relève de fonctions intellectuelles qui peuvent être extérieures ou non au champ scolaire et didactisées ou non. Le deuxième, culturel, est fait de savoirs et modes de connaissance généraux sur le monde non réductibles à la sphère scolaire mais donnant lieu tout autant à des classements et à des hiérarchisations. Le troisième est une composante identitaire symbolique, liée à ce que l'accès à un savoir requiert et construit un certain type d'identité personnelle et relie à une communauté pour laquelle il vaut.
Chacun des registres constitutifs du « scolaire » est lui-même mobilisable dans un régime « majeur » (littératié, élaboré) ou «mineur » (spontané, expérientiel). D'un point de vue cognitif, il peut renvoyer à des conceptualisations scientifiques ou du quotidien (Vygotski, 1997) ; d'un point de vue culturel, il peut référer à des savoirs et des pratiques « légitimes » ou, à l'inverse, "dominées » (Bourdieu, 1979) ; d'un point de vue symbolique, il peut relier à la communauté académique des savoirs verticalement structurés ou à des communautés plus proches, où les liens " horizontaux » supposent des savoirs moins rigoureux mais des identités plus incarnées (Bernstein, 2007). Or, dans l'école contemporaine, ces régimes mineurs et majeurs ne sont plus distingués en amont dans les programmes et, surtout, dans leurs modalités pédagogiques d'application. Il appartient donc aux élèves de s'inscrire dans chacun de ces registres en n'en confondant pas les frontières, les logiques internes et les dynamiques. Ils doivent, pour être dans une logique scolaire, adhérer à des manières de se tenir et d'apprendre spécifiques, mais aussi savoir que les façons d'apprendre à l'école ont en arrière-plan des savoirs robustes mais vivants, constitués selon des modalités différentes dont il faudra s'approcher. Ils doivent aussi comprendre que les savoirs, comme les pratiques auxquels ils se livrent, constituent des cultures différentes au sein de l'école et dans la société et enfin que l'adhésion aux valeurs qui unissent à d'autres suppose un équilibre subtil entre ses appartenances à l'école, à sa famille et au groupe des pairs. Une analyse plus complète de cette quadruple inscription est à faire. Il est cependant possible de l'esquisser en prenant en compte la double nature, diachronique et synchronique, des rapports entre ces différents registres de l'apprentissage.

\section{Un point de vue diachronique}

Une approche sociologique des savoirs, telle que celle inaugurée par Durkheim (1990), montre que les registres privilégiés par telle ou telle époque ne sont pas les mêmes. Le mouvement de massification du second degré en France a ainsi été concomitant d'une pénétration dans les curriculums scolaires de démarches critiques longtemps réservées à la sphère savante (Bourdieu \& Gros, 1989). La décompartimentation des apprentissages a promu des 
codes « ouverts » plus en phase avec des sociétés elles-mêmes « ouvertes» (Bernstein, 1967). Cette empreinte croissante du cognitif sur le scolaire s'opère elle-même sur fond de renouvellement du rapport au savoir d'une société qui voit s'effacer rapidement la frontière entre le profane et le savant en même temps que s'élever les exigences de leur maîtrise (Beck, 2001). Si la manière de faire école présente une certaine homogénéité à travers les âges, elle montre aussi des discontinuités, liées aux finalités éducatives mises en avant par une période historique et qui promeuvent ou délégitiment par exemple tel type de lecture ou d'écriture. (Rockwell, 2000, Chartier, 2005). La seule école maternelle française présente, depuis les années 1960, des évolutions rapides qui montrent les différents registres, cognitivo-scolaire d'une part et symbolico-culturel d'autre part, prendre tour à tour l'avantage (Bautier, 2006). Cette recherche d'équilibre entre registres, entre pédagogies visibles et pédagogies invisibles, semble particulièrement significative des « nouvelles classes moyennes ", tiraillées entre la recherche de l'épanouissement des personnes et celle de savoirs et savoir faire imposés par la réalité des rapports de classes.

\section{Un point de vue synchronique}

Ces quatre registres peuvent être regardés d'un point de vue synchronique selon l'équilibre relatif qui les constitue en configuration à un moment précis de l'histoire du système éducatif. Les élèves doivent apprendre à intérioriser les hiérarchies qui les ordonnent de manière modulable au long de leur cursus. Un élève comme Bassékou, étudié par Stéphane Bonnéry (2007), se trouve dans un important malentendu du fait qu'il ne parvient pas à comprendre que son adhésion symbolique à l'école (typique de nombre d'enfants de milieux populaires, a fortiori d'origine immigrée), récompensée en primaire car perçue comme une marque de bonne intégration, ne compense pas les ratés d'apprentissage qui sont déjà les siens en termes d'apprentissages scolaires et cognitifs et qui ne vont cesser de s'accroître avec la montée des exigences conceptuelles. Il découvre avec stupeur au collège que les manières de faire qui sont toujours les siennes ne conviennent plus. Il attribue ceci à l'arbitraire des adultes et en conçoit, comme beaucoup de collégiens dont les résultats chutent, un profond ressentiment à l'égard de l'école dont il risque de se détourner progressivement, incapable qu'il est de proposer des réponses autres qu'en termes de désaffiliation symbolique et culturelle. Or la réussite suppose au contraire un engagement toujours plus ferme dans les savoirs scolaires eux mêmes de plus en plus marqués par les exigences cognitives. Perceptibles sur des cas d'élèves et explicatives de leurs trajectoires, ces manières de configurer les registres d'apprentissage aident également à comprendre les identités et dynamiques d'établissements qui, selon qu'ils sont plutôt du centre ou de la périphérie, interprètent différemment les façons de faire tenir les scolarités (van Zanten, 2012 ; Bautier \& Rayou, 2013).

Il nous semble voir une analogie entre les métamorphoses de la culture (Coulangeon, 2011) et celles de la forme scolaire au sens où l' " omnivorité » qui caractérise notre époque ne signifie pas la disparition de la distinction sociale, mais une invisibilité plus grande de ses codes. La façon de comprendre et articuler la nature et le « poids » des différents registres constitue sans doute de ce point de vue un important discriminant, d'autant que les supports sur lesquels les élèves sont aujourd'hui amenés à apprendre et à s'exercer connaissent des évolutions qui ne leur facilitent pas la tâche.

\section{QU'EST-CE QU'UN TEXTE AUJOURD'HUI ? ÉVOLUTION CURRICULAIRE, DISPOSITIFS ET SUPPORTS}

Un élément caractéristique des dispositifs d'enseignement actuels, au moins de leurs supports, nous est apparu essentiel à prendre en considération (PISA en est justement emblématique) de façon plus précise que cela n'avait été fait dans nos précédents travaux pour l'étudier du point de vue des registres convoqués : il s'agit des évolutions curriculaires ${ }^{9}$ à l'œuvre dans les textes composites et non linéaires (discontinus) avec lesquels et sur lesquels les élèves doivent travailler. «La lecture d'un document composite implique de maîtriser aussi les codes propres à chaque composante sémiotique (image, graphique, texte, schéma...) ainsi que les codes permettant de mettre en rapport les différents documents les uns avec les autres - indications verbales (les légendes) ou non verbales (contiguité dans l'espace de la page, flèches, encadrés) des rapports entre ces compo- 
santes » (Bautier et alii, 2012, p. 65). Enfin, et c'est une autre caractéristique tant de ces documents que des situations de travail installées dans les classes, les non-dits concernant le travail cognitif et langagier à mettre en ouvre pour « lire » et effectuer le travail demandé laissent à la charge de l'élève son identification, alors même que l'ensemble de ce dispositif de travail est censé les conduire à la construction de savoirs nouveaux thématiques et génériques ${ }^{10}$.

La question soulevée par ce type de documents délinéarisés (ou discontinus dans la terminologie de PISA) et supports de travail est double. Elle porte sur l'évolution curriculaire qu'ils manifestent, mais aussi sur leur dimension aidante ou au contraire gênante quand il s'agit pour les élèves de les utiliser et de construire des savoirs à partir de leur « traitement ». En effet, «La souplesse d'utilisation de ces documents fragmentés et non linéaires, qui permet à leurs utilisateurs de choisir les informations dont ils ont besoin pour les aider à construire un modèle mental, a pour contrepartie une exigence cognitive plus grande, à la fois parce qu'ils sont moins guidés par une organisation rhétorique du texte et parce que les aspects métacognitifs liés à la régulation de leur lecture et la conscience de l'état de construction du modèle sont ici encore plus décisifs que dans la lecture d'un texte linéaire » (Bautier et alii, 2012, p. 65).

L'évolution curriculaire contemporaine, nous l'avons dit ailleurs (Bautier, 2010, Bautier \& Rayou, 2013, Bautier et alii, 2012), est marquée par un double enjeu cognitif et langagier. Enjeu cognitif quand il s'agit aujourd'hui davantage de savoir traiter cognitivement une hétérogénéité épistémologique, sémiotique et langagière, savoir produire explicitement pour la classe ou pour soi un « texte » oral ou écrit « homogène » car conceptuel, avec cette même hétérogénéité. Il s'agit dès lors pour PISA d'évaluer davantage des comportements cognitifs de mobilisation, de mise en relation, de tissage des savoirs et connaissances que d'évaluer, comme cela aurait été sans doute le cas il y a 30 ans encore, des connaissances acquises en situation scolaire et plus particulièrement des connaissances disciplinaires ${ }^{11}$. Mais, il faut avoir à l'esprit que, même si ces connaissances ne sont plus l'objet premier de l'évaluation, ne pas les mobiliser ne permet pas toujours d'effectuer les tâches proposées de façon satisfaisante ; on retrouve ici l'existence des registres scolaire, culturel, symbolique et cognitif évoqués précédemment, que l'élève doit savoir identifier et avec lesquels il doit savoir composer dans tous les sens du terme. L'évolution curriculaire va donc dans le sens d'une élévation des exigences à l'œuvre dans ces quatre registres et d'un implicite plus grand, même si, justement, il est possible et sans malentendu véritable d'effectuer ce qui est demandé sur le seul registre des connaissances personnelles. L'évaluation que font les enseignants des activités des élèves permet de parler de contrats pédagogiques différenciés, volontairement ou non (Rochex \& Crinon, 2011). La mobilisation du régime mineur des registres culturel, cognitif, symbolique, scolaire permet en effet à des élèves considérés comme faibles par les enseignants d'obtenir tout de même une évaluation moyenne.

L'enjeu est également langagier quand il s'agit de produire des textes à partir de ces documents. Dans ce domaine, on constate pourtant à l'heure actuelle et du fait des supports, des modes de travail et d'évaluation, un double paradoxe. D'une part, les documents comme les pratiques de classe ordinaires ne sollicitent que rarement les élèves pour des tâches d'écriture longue (les modalités actuelles d'évaluation par repérage, soulignements... influencent sans doute cette habitude); ainsi, le travail à partir de documents écrits se limite le plus souvent à du repérage ou à des réponses brèves, il en est de même du travail après observation ou manipulation, en sciences par exemple. D'autre part, les réponses sollicitées explicitement ne le sont qu'assez peu sur le registre conceptuel, générique et élaboratif, et bien davantage sur celui de l'opinion, de l'intériorité du sujet, ou de la description et du constat ponctuels. Le paradoxe vient du fait que les élèves, s'ils veulent dépasser les seules exigences formelles et ponctuelles, celles qui mettent en œuvre des savoirs segmentés (Bernstein, 2007), pour se situer dans l'univers des savoirs conceptuels que suppose l'appropriation-compréhension de savoirs génériques, doivent opérer sur le plan cognitif, langagier et linguistique un travail d'une grande complexité qui leur est rarement enseigné à l'école. Le modèle de compétence, par sa "générosité » sans doute (Bernstein, 2007), suppose en effet la plupart du temps cette compétence partagée qu'il suffirait donc, par des mises en situation adéquates, de mobiliser. Ce n'est cependant pas ce qui se produit.

Il s'agit, de la part des élèves, pour produire un texte à partir de documents discontinus et composites (documents de travail comme questions s'y 
rapportant), de les envisager comme un seul texte, d'y identifier ce qui fait le commun et la raison de les présenter ensemble, de construire à partir de cela un nouveau texte en utilisant généralement d'autres éléments plus conceptuels et génériques que ceux, langagiers et linguistiques, qui sont fournis. Ce texte, qu'il soit effectivement réalisé à l'écrit ou qu'il soit simplement intérieur, élément de réflexion, doit convoquer des éléments des textes présentés (qu'ils soient littéraires, scientifiques ou de vulgarisation médiatique ou un mixte de tout cela), les tisser ensemble, mais aussi reconstruire les éléments de connaissances et intentionnalités supposés faire partie du commun partagé et qui ne sont pas explicités (régime majeur du registre culturel ). Il s'agit alors de mettre en mots ce qui ne l'est pas, de combler les vides (le rapiéçage), de solliciter les savoirs et connaissances non contenus dans le texte et pas toujours scolaires. Il est nécessaire pour l'élève de s'autoriser à prendre les textes au sérieux et à les travailler pour eux-mêmes (et pas « seulement » en faire des supports de l'expression de sa réaction, de son opinion et de son expérience, mais aussi à aller bien au-delà des textes).

Mais avec quelles consignes et ressources pour ce faire, avec quelle socialisation scolaire ou familiale pour penser ces possibles non dits ? Là résident pour de nombreux élèves des difficultés qui leur sont parfois invisibles à eux-mêmes. Dès lors, les productions des élèves dits fragiles oscillent entre des textes d'intériorité et d'expression et des textes de formalisme scolaire ponctuel. Dans les deux cas le travail effectué ne relève que d'une dimension mineure d'élaboration et de conceptualisation. Autorisés par les consignes et supports proposés, des contrats didactiques différenciés se mettent ainsi en place. La méthodologie adoptée pour cette recherche (faire passer les épreuves PISA à des élèves de 15 ans, en troisième ou en seconde, puis, lors d'un entretien, revenir avec chacun d'entre eux sur leurs réponses afin d'accéder aux logiques qui les sous-tendaient) a permis de caractériser des modes de faire assez généraux dans ces situations à caractère scolaire. C'est ce caractère récurrent des manières de faire face aux textes chez une quarantaine d'élèves d'établissements différents qui nous autorise à décrire des modalités de réponse générales ou typiques. Ces modalités permettent de faire des hypothèses sur les causes des difficultés scolaires de certains élèves

\section{L'exemple de « Guide de conduite »}

Ce texte délinéarisé (voir Annexes), donné sous forme de liste aux élèves testés par PISA, fait l'objet de questions qui nécessitent un important travail de tissage de la part de ses lecteurs. Il est certes encadré par des marqueurs chronologiques présents dans les conseils 1 : « le point de départ d'une année scolaire », 13, « la fin de l'année » et 14 « une fois rentré chez vous ». Mais pour le reste, la trame n'est pas explicitement chronologique. Plus exactement, se substitue à la chronologie d'organisation du séjour celle des relations entre diverses identités de l'étudiant qui y participe : une identité nationale emportée avec soi et qui en rencontre une autre; une identité personnelle mise à l'épreuve de cette confrontation ; une identité ultérieurement enrichie par ces rencontres. Mais les injonctions à assumer positivement ces relations ne suivent pas forcément cette chronologie, à preuve les points 2 et 7 , par exemple, qui sont des considérations très générales sur l'importance d'être soi.

La note de bas de page donne une clé de lecture de ce texte en le contextualisant. Y accéder suppose une compétence non-linéaire de lecture (vs « en file indienne ») qui consiste à aller du titre au bas du texte avant même d'être entré dans celui-ci. La mise en forme du texte est donc organisée par une réflexion sur l'identité qui ne peut être perçue que si les différentes injonctions portées par les nombreuses tournures impératives sont lues comme des variations sur la thématique de l'échange et du changement. Les élèves rompus aux techniques du traitement de texte peuvent en trouver une autre clé dans la mise en gras de certaines de ses injonctions qui émargent au triple registre de l'identité : d'originaire d'un pays, de jeune mis à l'épreuve, de personne travaillant pour des bénéfices à long terme. Les questions posées sur le texte le sont par le biais d'un QCM dont les élèves français sont assez peu familiers et dont les logiques qui sous-tendent les propositions ne sont souvent, dans PISA, pas les mêmes d'une question à l'autre.

La question 18 appelle une réduction du texte à son intention principale (« ce guide insiste surtout sur l'importance ». Mais cet appel à repérer l'essentiel reçoit des réponses dans l'examen du texte. Le texte est donc référé à lui-même selon une logique en jeu de l'exactitude. Le nombre d'occurrences relatives aux relations entre les autres et soi doit faire pencher pour la réponse $\mathrm{D}$, la plus générique. Il est assez facile de montrer que des réponses comme la $\mathrm{A}$ et la $\mathrm{B}$, quoique 
plausibles, ne rendent que partiellement compte de l'ensemble. La réponse $C$ n'est, quant à elle, appuyée par aucun élément du texte (sauf à faire une confusion sur la signification du point 14). Un comptage des propositions compatibles avec ce que contient la réponse D doit suffire à départager les items du QCM.

La question 19 appelle une autre logique de mise en rapports entre le texte et le nouveau texte que devient la réponse proposée. Il ne s'agit plus en effet de trouver des correspondances terme à terme entre la question et le texte, mais de se prononcer sur une vraisemblance. Il faut en quelque sorte accepter de sortir de la littéralité du texte pour évaluer une proposition qui aide à l'interpréter. Portant en apparence sur un point particulier du texte, celle-ci aide en réalité à en saisir un des organisateurs profonds qu'est l'affirmation de la haute valeur de la singularité des personnes. La réponse A constitue une tentation réelle puisqu'elle se réfère à des arguments du texte, mais pas à la suggestion à évaluer. Les réponses $C$ et $\mathrm{D}$ sont soit à contre-sens, soit hors-jeu et renvoient à la logique de l'exactitude qui n'est pas au cœur de cette question.

La question 20 semble relever du genre de l'hypothétique car le contexte auquel elle se réfère est celui de sa portée pour la personne qui a effectué un échange "positif ». Il n'est pas possible d'y répondre à partir d'éléments présentés par le texte lui-même, ni de restituer sa philosophie sous-jacente. Il faut imaginer ce que deviendra l'identité du voyageur qui a dû affronter l'autre et qui, de retour chez lui, est confronté à l'altérité que constitue désormais un univers autrefois familier. La sortie du texte-support, la confrontation avec des suggestions d'interprétation proposées par le texte-questions supposent une incursion dans l'hypertexte d'une réflexion philosophique sur la dialectique de l'identité. Le « cela ne fait que commencer » montre clairement à quel point il faut, pour comprendre le texte, ne pas confondre le commencement temporel (le début de l'année scolaire, et la tentation que constitue la réponse $\mathrm{A}$ ) avec l'étape de développement de soi que constitue un retour qui est tout sauf un achèvement (il faut alors refuser la tentation de la vision cyclique de la réponse $\mathrm{B}$ ). La réponse $\mathrm{C}$ se situe bien, quant à elle, dans le registre de la chronologie identitaire, mais elle en propose une version non dialectique qui renvoie à une étrangeté des choses (« un environnement inconnu ») et non pas à la modification de « la vie» opérée par la mise à l'épreuve de soi dans l'échange.

\section{Des registres de compréhension}

La compréhension du texte suppose, outre ce travail de tissage, voire de rapiéçage, une aptitude à se déplacer dans les quatre registres précédemment définis.

\section{Registre scolaire}

Guide de conduite propose une situation qui ne relève pas explicitement du registre scolaire, car les trois QCM qui le constituent ne sont pas très présents dans les pratiques de classes d'élèves français. Bien qu'inscrite dans un contexte a-didactique, la question 18 renvoie à des acquis scolaires transférables sur du non scolaire, comme lire un texte en entier avant de répondre à des questions, le contextualiser ou ne pas prendre la partie pour le tout. Pour savoir ce sur quoi insiste le texte proposé il faut aussi avoir l'habitude de discriminer ce qui est un moyen (représenter son pays, se faire des amis, avoir de bonnes relations avec la famille d'accueil) et ce qui est une fin (vivre une expérience enrichissante pour soi et pour les autres et non pas se situer comme le représentant impersonnel de son pays à l'étranger).

Parmi les élèves qui donnent des réponses fautives figurent ceux qui n'ont pas eu une approche synthétique du texte et l'ont lu soit en en prélevant arbitrairement des éléments (lecture segmentée), soit en le lisant in extenso mais en manquant l'appel de note qui permettait de le contextualiser. C'est le cas de Narmada (3e) («Ah non j'ai pas vu ! ») qui a commencé à vouloir répondre sans prendre connaissance de l'ensemble. A la fin de notre entretien, elle n'a « toujours pas compris » ce qu'est un programme d'échange. D'autres se disent surpris par la forme, ainsi Antoine (3e) : « j'ai eu des questionnaires de ce genre, mais pas beaucoup », ou Morgane (3e) qui se dit surprise par la demande de « donner son avis». Ceux qui fournissent de bonnes réponses sont davantage capables de rapprocher l'exercice de PISA de ce qu'ils font en classe : «En fait c'est comme si je faisais mon programme de troisième... C'est une répétition en fait, puisque j' lis les textes, après on m'pose des questions dessus, après j'analyse mon texte pour permettre de trouver les réponses ». (Amandine (3e). Attia, (2nde), qui a fait un sans faute, connaît son défaut et le combat: " c'est que je lis pas le texte directement, j'essaie pas de comprendre tout d'abord avant de répondre aux questions, c'est... je me jette 
d'abord sur les questions et après j'essaie de voir les réponses dans le texte, c'est ça mon défaut ».

\section{Registre culturel}

Cet exercice requiert assez peu de connaissances générales sur le monde puisqu'il ne vise pas un type particulier de séjour à l'étranger, il en nécessite néanmoins sur le rôle de dispositifs péri-scolaires comme les échanges linguistiques. Il est en effet difficile d'envisager les bénéfices supposés d'une telle pratique si on l'ignore et si on n'imagine pas les types de complémentarité qu'elle entretient avec les apprentissages scolaires. Il s'agit donc de sortir des frontières de l'école au sens strict du terme pour se projeter non seulement au dehors d'elle mais aussi dans un avenir un peu lointain, celui où on sera « étudiant » (pour un jeune français de 15 ans, cela ne peut signifier que le post-bac).

L'essentiel des lacunes relatives au registre culturel se situent dans la méconnaissance par les élèves de notre échantillon, qui, pour beaucoup, ne franchissent que rarement les frontières de leur quartier, des programmes linguistiques. Narmada pense que ces séjours « c'est du genre internat non? » et ne comprend pas le rôle des familles d'accueil. Laurent (3e) devait aller en Angleterre avec sa classe, mais ( " ma moyenne elle était trop mauvaise, alors j'y ai pas été ») ne sait pas trop de quoi on parle. Antoine a lui aussi été désarçonné : "Moi, au début, j’ai trouvé, j'me suis dit que c'était... j'ai pensé que c'est une blague, parce que, moi., j'sais pas... »programme d'échange «, j'sais pas si ça existe ! »

\section{Registre cognitif}

Les compétences cognitives requises sont en revanche nombreuses. A commencer par la compréhension de ce qu'un même format de QCM sert à répondre à des questions qui mettent en jeu des valeurs de vérité différentes (l'exact, le vraisemblable, l'hypothétique). Plus que la question 18, les questions 19 et 20 portent davantage sur l'esprit que sur la lettre et mettent en jeu des raisonnements qui ne se résorbent pas dans des procédures scolaires. La question 19, en proposant non pas un élément du texte en tant que tel, mais un autre donné comme équivalent, ne fait plus travailler sur la mise en correspondance entre une réponse possible et le texte, mais sur le rapport entre une assertion externe et celui-ci. Elle suppose une autonomie cognitive supérieure à celle que procurent les routines argumentatives. La réponse $B$ ne peut s'imposer que parce que, une fois éliminées $\mathrm{D}$, dont la généralité est hors de propos ainsi que $\mathrm{A}$ et $\mathrm{C}$, qui proposent deux versions antithétiques et exagérées du rapport entre soi et les autres, la proximité de sens avec l'intention globale du texte peut être établie. La question qui porte sur un prolongement de l'expérience propose quant à elle trois réponses parfaitement vraisemblables (A, $\mathrm{B}, \mathrm{D})$ et une relativement plausible (C) mais dont la pertinence est inégale si on la réfère à la dialectique du même et de l'autre qui sous-tend tout cet exercice et que condense la seule réponse $\mathrm{D}$.

La question 20, par le saut qu'elle opère hors du procédural scolaire vers le cognitif, déroute beaucoup de répondants. Si Worokia (3e) élimine une mauvaise réponse (A), c'est de façon chanceuse pour de mauvaises raisons empruntées à sa propre scolarité : "J'peux pas répondre la $\mathrm{A}$, parce que c'est toujours une année scolaire, parce que chaque année c'est toujours la même chose. Pour moi, si j'passe, ce s'rait un nouveau environnement, parce que j'serai au lycée, c'est nouveau. J'connais pas les gens et les professeurs, alors qu'ici, si j'avais répondu la question $\mathrm{A}, \mathrm{y}$ a quelques-uns des professeurs que je connais». L'idée selon laquelle « cela ne fait que commencer » (une nouvelle expérience identitaire et non pas une nouvelle année scolaire) ne survient pas facilement. Car rien dans le texte stricto sensu ne permet d'y répondre et le recours à l'expérience propre se révèle réducteur pour des élèves qui, précisément, n'ont jamais bénéficié d'un tel dispositif d'échange. Certains élèves, comme Youssef (2nde), font en revanche un sans faute sur l'exercice car ils comprennent que, malgré le caractère inhabituel de celui-ci, «c'est presque pareil que ce qu'on fait, généralement $»$. Maîtrisant le procédural scolaire, ils parviennent à articuler les exigences de renvoi au texte et celles d'élaboration personnelle (« Ils nous disent de relever des informations du texte, et après on essaie d'argumenter avec nos mots »), à établir entre des disciplines scolaires qu'ils maîtrisent des relations transversales qui lui confèrent les compétences nécessaires ( Ben, déjà en cours de français, les études de texte. En histoire aussi, les études de documents $»$ ).

\section{Registre symbolique}

Les thématiques du texte comportent de nombreuses références aux identités des participants au programme d'échange (Être soi-même au milieu 
d'autres (2) ; être soi-même à l'aide d'autres (9); créer de la réciprocité (11); entretenir des réseaux (14). Elles impliquent, pour être saisies, une labilité identitaire de la part du lecteur qui, sinon adhère, du moins admette une philosophie du développement de soi par l'échange avec des étrangers, l'affirmation de sa différence au milieu d'autres, la recherche de l'épanouissement personnel, le lien entre les opportunités scolaires et celles qu'offre l'expérience juvénile, etc. qui n'est pas nécessairement partagée par tous les jeunes scolarisés souvent adeptes de comportements conformistes au sein des groupes de pairs. Peu explicitée par le Guide, cette thématique y est cependant structurante et son repérage est décisif pour répondre correctement aux questions 19 et 20. Elle est vraisemblablement la trame profonde qui permet de « re-linéariser » les 14 conseils et de réussir l'exercice.

Or les jeunes soumis à cet item commettent de nombreux contre-sens sur la vision de l'autonomie personnelle proposée par le Guide. De nombreux contenus doxiques apparaissent dans les réponses, inspirés notamment par les comportements juvéniles en termes de "tyrannie » de la majorité (Pasquier, 2005) qui font estimer, à contre-sens de ce que dit le texte, que ne pas être comme tout le monde «ne rend pas forcément plus intéressant $»$ (Lucie (2nde). De même les préconisations du Guide ne sont pas tant interprétées comme des conseils que comme des « ordres ». Or « Les gens savent ce qu'ils doivent faire » (Antoine, 3e). Warda (3e) estime comme lui que « ben, oui, normalement, je crois qu'ils disent : "faites comme vous voulez", mais, hein, [...] carrément, ils dictent ce qu'il faut faire, donc eh ! ». Lorsque la doxa partagée ne s'impose pas, c'est l'expérience personnelle qui peut prendre le relais. Le sens d' "échange " peut ainsi n'être pas compris quand la non pratique de cette activité extra-scolaire se joint à la centration sur des problèmes « psychologiques » pour en produire un sens qui n'est pas celui du texte. Pour Clément (3e), « "n'hésitez pas à demander conseil", ça veut dire que quand on a un problème on doit pas garder ça pour nous tout seul, on doit le dire aux autres ». Outre la maîtrise des techniques scolaires, la connaissance des échanges linguistiques, l'accès au cognitif requiert une maîtrise des identités multiples mises en jeu par le texte. Umba, élève de Seconde, se hisse à ce niveau en explicitant leur dialectique : elle comprend bien que « si on va dans un autre pays, c'est pas que pour s'im- poser, pour dire, euh, par exemple "en France c'est comme-ci, c'est comme ça, je veux manger que ci, je veux manger que ça", mais que, en même temps, on est là pour apprendre une nouvelle culture, on ne doit pas oublier qui on est nous, on doit pas dire, "bon, j'deviens comme ils sont en Angleterre et j'deviens anglais", non, on est français quelque part, il faut pas l'oublier ça ».

«Guide» nous semble un bon exemple de ce que nous entendons par « registres» et de la façon dont ils sont mis en jeu par le travail scolaire sur des textes. L'analyse de l'exemple suivant se propose de montrer plus finement les enjeux des mobilisations «majeure » et « mineure» de ces registres.

\section{L'exemple de Macondo}

Nous avons également choisi l'exemple de ce texte (voir Annexes) de l'évaluation PISA 2000, un des rares textes mentionné par les élèves comme le plus proche du connu, sans doute parce que littéraire et donc relevant du « scolaire », car il met en évidence par sa nature même et les questions posées aux élèves et, indépendamment donc de son apparente continuité, la pluralité des registres de travail que peuvent et que doivent convoquer les élèves pour effectuer les tâches demandées. Il permet aussi de mettre en évidence l'écart qu'il peut y avoir entre les réponses minimales et possibles de certains élèves et le travail de mobilisation cognitive et culturelle que peuvent effectuer d'autres élèves et qui leur permet de " comprendre » le texte, tout au moins d'en construire une signification correspondant aux réponses attendues.

«Macondo » est un extrait de 16 lignes en un seul paragraphe issu du roman de Gabriel Garcia Marquez, Cent ans de solitude. La langue, par sa syntaxe (inversions des compléments et des adjectifs, emploi du passé simple, relatifs composés) et son lexique, est très difficile d'accès et oblige sans aucun doute à plusieurs lectures si on veut s'approprier le texte, « entrer dedans ». Ce texte met les élèves dans une situation de malaise et d'incompréhension car si, dans un premier temps, ils se sont crus en « terrain familier », ils se sont, pour un grand nombre d'entre eux, aperçu assez rapidement que le texte était « étrange », " très difficile », « bizarre » et qu'ils ne comprenaient pas grand chose. Incontestablement, il n'est pas de ceux que « fréquentent » les élèves, même 
en classe de troisième ou de seconde. On peut d'ailleurs aller jusqu'à le qualifier de discontinu, comme d'autres textes de PISA, dans la mesure où l'extrait du roman n'est pas « auto-suffisant » car seule la mise en relation du texte avec le «paratexte » permet d'en approcher le sens et le contexte culturel qui éclaire les formulations. Ce «paratexte» est ici constitué par la consigne, la précision qui l'accompagne (dans une syntaxe d'ailleurs assez étrange - : « Dans cette partie du récit, on vient d'introduire le chemin de fer et l'électricité dans la petite ville imaginaire de Macondo, et d'ouvrir le premier cinéma ») et par l'ensemble des questions posées.

$\mathrm{Du}$ point de vue de l'énonciation, le texte est également faussement linéaire car les points de vue sur la situation sont énoncés sans explicitation par des personnes très différentes : l'auteur du roman, les différents personnages.

Les questions, au nombre de quatre, sont de trois types:

- La première question est de type "repérage d'information » (Dans les films, quel est l'élément qui a mis en colère les gens de Macondo ?) elle peut donc être comprise sur le régime mineur du registre scolaire ; nombreux sont d'ailleurs les élèves qui recopient la question avant de la compléter par la phrase relevée, comme le demande l'école (faire une phrase complète). Ce type de question est celui qui conduit les élèves à recopier une ou deux phrases du texte contenant la cause de la colère. Il est à noter que les plus faibles recopient la cause telle que formulée par les gens de Macondo et très contextualisée (« que l'acteur qu'ils avaient cru mort réapparaisse dans un autre film bien vivant $»$ ), d'autres relèvent une phrase plus générale ( « Don Bruno Crespi a fait une proclamation disant que le cinéma n'était qu'une machine à illusion »).

- Les deux questions suivantes proposent sous forme de QCM quatre réponses possibles visant à vérifier la compréhension de l'état d'esprit des gens de Macondo. Globalement, les élèves sélectionnent les bonnes réponses. Pour la question 3, on peut encore mobiliser essentiellement le registre scolaire car une des phrases du texte est proposée parmi trois autres possibilités, et c'est évidemment cette proposition qui est choisie par les élèves en très grande majorité. Concernant la question 3 , en $3 e$ et plus encore en 2nde, ils font tous la différence entre acteurs et personnage et donc convoquent aisément ce registre d'une connaissance scolaire enseignée très tôt et choisissent cette réponse la plus adéquate étant donné le contexte.

- La quatrième question ( « Êtes-vous d'accord avec le jugement final des gens de Macondo à propos de l'intérêt du cinéma ? Expliquez votre réponse en comparant votre attitude et la leur à l'égard des films ») est celle censée susciter la production d'un texte long, six lignes sont prévues pour cela et plus si nécessaire.

L'ensemble des trois premières questions peut être considéré comme mettant l'accent sur la construction par les gens de Macondo d'un rapport spécifique au cinéma : celui de gens qui ne savent pas ce que c'est, qui y voient « un documentaire», qui n'ont pas « encore» construit la différence entre l'acteur et le personnage. Pour répondre de façon pertinente à la quatrième question, sans doute fallait-il donc la mettre en relation avec les trois autres ainsi qu'avec l'information initiale de la consigne générale qui contextualisait historiquement le passage du roman. Mais cette démarche suppose de lire de manière non « segmentée », de s'éloigner d'une lecture au " pas à pas », de produire un travail cognitif de mise en relation, ce que la présentation des questions n'implique pas a priori et qui est une forme de lecture peu fréquente chez une grande partie des élèves. Nous avions déjà souligné ce mode de lecture segmenté (Bautier, 1989) qui ne construit pas le texte comme texte chez les jeunes en difficultés scolaires.

La formulation de la question devrait donc conduire l'élève à se situer simultanément sur le registre symbolique de l'avis personnel concernant les goûts cinématographiques et le rapport au cinéma, donc d'avoir un choix à faire, conscient ou non, (faut-il aller au cinéma pour se distraire et oublier la vie quotidienne, ou parce que le cinéma est un art important... ), et sur le registre culturel les conduisant à la prise de distance à l'égard des positions des gens de Macondo dont c'était la première expérience cinématographique. Certes, il est possible d'obtenir des points à l'évaluation en répondant avec son seul rapport personnel au cinéma et en laissant dans l'oubli les gens de Macondo, (la majorité des élèves en $3^{\mathrm{e}}$ et en ZEP ont répondu ainsi ${ }^{12}$. Certes, il est également possible de répondre qu'on aime se distraire et oublier les soucis en regardant des films d'horreur ou des blockbusters. Mais les meilleures évaluations vont aux élèves qui SE mobilisent dans 
la circulation entre les registres; ils interprètent la situation non seulement comme une interrogation sur leurs goûts personnel, mais comme l'occasion d'une réflexion générale sur le cinéma dans laquelle ils s'inscrivent, sans doute à partir de leur expérience du cinéma, mais en se mobilisant au-delà de celleci, en la travaillant pour la (re)penser au-delà d'ellemême. «Dans le premier cas (celui des élèves en difficultés), l'expérience sensible est seulement appelée à être, au mieux, partagée, au moins communiquée, dans l'autre, elle est reconfigurée. C'est au prix de ce travail que l'élève est de plain-pied avec les exigences scolaires. Celles-ci ne sont pas réductibles à un arbitraire culturel, elles sont proches de la première acception de « subjectivité », celle qui est à mettre en relation avec la notion de sujet émancipé participant de la communauté scolaire de l'écrire (parler)-penser avec des savoirs (ou ce qui est construit comme tels) où l'activité langagière est peu dissociable de l'activité de connaissance » (Bautier, 2005).

Cependant, la valorisation de la production d'un texte complexe, de tissage, de reconfiguration et mobilisation de l'hétérogénéité des registres pour se faire penser au-delà du texte donné n'est pas un simple instrument de domination culturelle. Bien au contraire, il est possible de penser la démocratisation de l'école, l'égalité, comme la possibilité pour chacun de s'émanciper de ses propres avis ou goûts, de pouvoir se situer pour cela dans une dimension historique, géographique ou culturelle.

\section{Ce que font les élèves avec ces textes}

\section{Registre cognitif: Quand la lecture est segmentée et} « trop » scolaire

Le texte de Garcia Marquez n'a pas bloqué complètement les élèves dans leurs possibilités de répondre, même de façon erronée. Sans doute est-ce parce qu'il a été jugé le plus familier scolairement parlant du fait que c'est un texte littéraire. En revanche, les textes plus composites et discontinus leur posent souvent des problèmes insolubles : si les enseignants ne les aident pas, les élèves voient difficilement ce qu'ils peuvent faire avec de tels documents. Bien qu'il s'agisse de textes non scolaires, ils sont encore plus étrangers pour les élèves qui ne semblent guère dans la familiarité avec les textes écrits quels qu'en soient le genre et le support (ils le disent explicitement). L'idée même qu'un texte puisse entraîner question- nement, réflexion, travail donc semble décontenancer ces élèves beaucoup plus familiers des textes scolaires « traditionnels ». D'ailleurs, les documents proposés par les manuels, par exemple en histoire ou en sciences, ne semblent pas leur permettre de travailler, sans doute pour les mêmes raisons : ils supposent comparaison, réflexion et mise en relation au-delà d'eux-mêmes et surtout raisonnement, c'est-à-dire déduction, hypothèses, conceptualisation, construction d'un nouveau savoir ou d'une nouvelle connaissance. La construction du sens par la mobilisation et la mise en relation des différents éléments textuels et non textuels n'est pas une pratique qui semble possible ou plutôt imaginable pour eux : le sens est soit donné littéralement dans le texte, soit incompréhensible, mais pour les élèves, il ne peut supposer un travail de la part du lecteur.

Une première remarque très générale permet de penser ensemble la notion de discontinuité du texte et celle de registre cognitif plus spécifique, le registre cognitif littératié : aucun des trente élèves de troisième (donc ayant déjà redoublé une classe) n'a mis en relation les éléments mentionnés ci-dessus. Dès lors, aucun d'eux n'a pu identifier le contexte de l'extrait du texte et donc n'a compris l'argument global. Cela ne les empêche nullement de répondre ponctuellement à des questions, mais évidemment sans tenir compte des liens qui les unissent. Ce type de lecture peut sembler paradoxal, car si les élèves s'en tiennent à des mots du texte, ne mobilisent que ces mots, ne "sortent » pas du texte en mobilisant des connaissances pour l'interpréter, ils ne prennent pas non plus au sérieux les éléments de la langue ( en particulier, les connecteurs, les pronoms, les temps des verbes...) qui permettent d'en construire au moins le sens littéral (ainsi c'est « le film qui se moque des spectateurs »' alors que le texte mentionne explicitement que les gens de Macondo ont pensé que c'est celui qui montre le film qui se moque d'eux, d'où leur colère).

Cette lecture «segmentée », qui peut correspondre à une "sur-attention » à des sortes de mots signaux qui font particulièrement sens pour les élèves, peut aussi correspondre à des habitudes de lecture construites très tôt dans la scolarité. L'habitude scolaire de repérage, suite aux questions de compréhension et d'évaluation dans l'ordinaire des classes dès la maternelle, semble perdurer pour les élèves en difficulté. Ici, ils repèrent sans problème tous la « bonne » phrase pour répondre à la question 
1, sans la comprendre ni l'utiliser ensuite dans un raisonnement pour répondre aux autres questions (le mot commun à la question et au texte suffit d'ailleurs pour trouver la bonne phrase). On voit ici que la distinction entre les régimes « majeur » et « mineur » des registres est heuristique. En effet, les élèves qui nous occupent sont « encombrés » par des représentations des attentes et savoirs scolaires figés et strictement formels qui les empêchent de se mobiliser eux-mêmes sur ce qu'ils parviennent pourtant à construire avec l'étayage des demandes de précision du chercheur. Ce régime mineur du registre scolaire qui n'est plus vivifié par des savoirs et de l'élaboration, semble en fait empêcher la réflexion puisque répondre est plus important que « bien répondre ». On peut encore prendre pour exemple de réaction « trop » scolaire et qui devient bloquante, celui de Mendy, en difficulté pour répondre car elle voudrait faire, " comme d'habitude », un résumé - ce qui n'est pas demandé - mais s'en trouve empêchée par la complexité du texte.

La nécessité de mobiliser des raisonnements et connaissances au-delà du texte n'est pas considérée, lors des entretiens, par ces élèves comme un comportement envisageable ni souhaitable scolairement. Les expressions du type "j'ai noté ça comme ça », « je lis pas beaucoup les questions, en fait $»$ sont très fréquentes. Il faut évidemment relativiser ces assertions du fait de l'énorme difficulté qu'ont ces élèves à justifier, à mettre en mots leurs propres manières de faire, leurs opinions et raisonnements, à mettre en mots simplement... Mais, on peut mettre ce type de remarques en relation avec celle de Priscilla : « quand on sait pas, on invente». Ainsi, répondre avec « un petit morceau du texte » ou un mot, celui qui a été compris, même s'il ne correspond pas «vraiment » à la question ne semble pas un comportement incongru car les questions sont, pour ces élèves, faiblement contraignantes. On peut rapprocher ce comportement de cet autre : une compréhension très parcellaire et approximative dont les élèves sont conscients sans en paraître gênés puisque l'important est de répondre quelque chose dans l'immédiateté de la succession des questions.

\section{Quand les régimes mineurs du symbolique et $d u$ cognitif se rejoignent.}

Si un mot, un morceau de phrase compris suffisent pour écrire une réponse, ils suffisent également pour construire une interprétation du texte, pour « dériver » sur le registre symbolique personnel, autrui ne pouvant être qu'identique à soi, à partir de ce mot ou morceau de phrase et interpréter en fonction « de ce qui se rapproche le plus de ce que je pense ». C'est ainsi que procèdent Warda, 3e, qui avait pensé que les «gens de Macondo voulaient rigoler ${ }^{13}$ », ou Laurent, $3 \mathrm{e}$ : « j'imagine sans chercher dans le texte ». Le registre scolaire de la lecture littératiée n'est pas convoqué ici car lire un texte, c'est réagir, se raconter des histoires indépendamment de la réalité du texte. Ce mode de lecture est une constante chez ce type d'élèves qui distinguent lire un texte et le comprendre (Bélenguier, 2009).

Ces élèves convoquent et érigent en norme le seul registre symbolique : leur réalité et leur valeurs sont LA réalité et LES valeurs que tout le monde ne peut que partager indépendamment des temps et des espaces. Il ne peut donc y avoir d'interprétation du texte. Le fictionnel est vrai ou faux lui aussi ; ce que disent les spectateurs de Macondo est donc « faux». Certaines des opérations cognitives ne semblent dès lors pas réalisables, il en est ainsi de la comparaison, qu'il s'agisse de comparer deux textes, deux avis, deux faits ou de se comparer aux autres. Ainsi, tous les avis d'élèves (dans une autre question de PISA qui juxtapose des opinions sur la conquête spatiale) sont identiques : « ils parlent tous de l'espace », ou dans Macondo : «ça parle de gens qui vont au cinéma » (Priscilla) (c'est aussi cela l'approximation). La tension entre les logiques sociale (registre symbolique) et cognitive (registre cognitif) est très (trop) grande pour certains élèves : « j’ai pas compris la question-concernant la comparaison entre l'attitude des gens de Macondo et celle des élèves quant au cinéma- je peux pas comparer par rapport à moi parce que moi c'est maintenant ». Il en est de même de l'activité de poser des hypothèses, qu'il s'agisse d'hypothèses sur des objets du monde ou des hypothèses interprétatives des textes, ou la pensée du général, ici, l'approche du cinéma et non tel film en particulier.

Quand le registre culturel ne peut (alors) qu'être mobilisé sur sa logique "basse ": ramener

l'inconnu au connu

L'absence d'hypothèses (registre cognitif), de possibilité de sortir de soi (registre symbolique) et du texte (registre scolaire), de connaissances sur le monde (registre culturel) conduit à ramener l'inconnu à son propre univers. Il peut s'agir de celui de la « télé-réalité » comme le disent certains élèves 
qui pensent que les gens de Macondo y sont, comme eux, confrontés, car ils ne peuvent imaginer un monde sans cinéma ou télévision. Pour expliquer le refus d'aller au cinéma chez les gens de Macondo, les réponses peuvent faire référence à un monde où l'argent manque pour aller au cinéma ou celui dans lequel les Arabes ne sont pas aimés, un monde bien proche du leur, de leur expérience, le mobiliser est pour la plupart des élèves une réaction spontanée.

En conclusion, si PISA et l'école d'aujourd'hui supposent la mise en relation de connaissances et savoirs scolaires et non scolaires, supposent de savoir utiliser et hiérarchiser les différents registres, à l'évidence ces élèves n'y parviennent pas. Dès lors, catégoriser ce qu'ils mobilisent en termes de savoirs ou de connaissances absentes n'est pas véritablement pertinent.

\section{Comment font les élèves plus en phase avec les attentes scolaires}

On se trouve ici face à des comportements à l'opposé de ceux que nous venons de décrire.

Chez les élèves de seconde, le régime majeur du registre scolaire est très prégnant : ils jugent le texte difficile mais ils inscrivent leur lecture dans une volonté de le comprendre avec tous les moyens dont ils disposent. Ils le lisent et relisent, retravaillent une première compréhension qui leur semble erronée. TOUS ont lu les précisions apportées sur le contexte, les questions, comme permettant de construire la signification globale et le plus souvent une signification générique ( «ils n’ont pas compris la magie du cinéma », Asmahani, 2nde). La mobilisation d'un registre culturel est présente chez certains. Pour pouvoir interpréter les réactions des spectateurs au-delà du texte, ils contextualisent historiquement la situation : «c'est la création du cinéma et les gens de Macondo n'étaient pas cultivés " (Samir, 2nde) sans s'attacher à la spécificité du film dont il est question. Certains élèves, toujours dans une volonté interprétative du comportement de colère des gens de Macondo mobilisent certes le régime mineur du registre culturel en faisant référence à des hypothèses qui les concernent. Cependant, à la différence des élèves de $3^{\mathrm{e}}$, il suffit d'une demande du chercheur lors de l'entretien sur l'origine de l'hypothèse pour que l'élève révise immédiatement la proposition et revienne au travail sur le texte

\section{CONCLUSION}

La démarche d'analyse des situations de travail et des activités des élèves présentée ici nous semble permettre de mettre en évidence les logiques hétérogènes qui les sous-tendent et de préciser ainsi ce qui, dans ces logiques, est cause de difficultés ou est manière de faire favorable au développement de nouveaux apprentissages.

Les évolutions curriculaires décrites précédemment sont évidemment en phase avec celles des relations entre la société et son École. Car la montée des exigences en compétences hautement littératiées va de pair avec l'effacement de la clôture symbolique, des frontières entre disciplines comme celles qui les distinguent des savoirs de la vie ordinaire, sur laquelle l'École française s'est construite. Dès lors, la mobilisation des registres culturel, symbolique et cognitif, autrefois ouverte à qui avait des curiosités qui excédaient les exigences scolaires, devient une nécessité pour tous. Une telle élévation des attentes, insuffisamment accompagnée par l'institution pour des élèves peu disposés à circuler entre ces registres, semble largement responsable de leurs difficultés et des différences d'apprentissage qu'ils accumulent avec les autres.

Ainsi, la description en termes de malentendus et surtout de registres mobilisés dans leurs deux régimes majeur et mineur apparaît heuristique pour mettre au jour des phénomènes peu apparents dans les productions écrites ou orales des élèves. En effet, la mobilisation de ces différents registres sollicités de façon implicite par les situations et documents, et réalisés de même plus ou moins consciemment par les élèves, revient pour ces derniers « à faire avec euxmêmes » comme ressources. Or ces ressources culturelles, symboliques, cognitives sont fort diverses dans leurs dimensions comme dans leur complexité. Car, par-delà le constat assez banal que les élèves en difficulté ou en échec ne répondent que très imparfaitement aux attentes de l'école, il est possible d'accéder à des profils d'élèves à partir de la configuration qu'ils établissent entre les registres et, supposons-nous, de les aider plus efficacement à progresser. Il se peut ainsi qu'ils n'identifient pas les registres à convoquer -ou s'interdisent de le faire-, qu'ils les hiérarchisent incorrectement lorsqu'ils les repèrent ou encore que leur compréhension « mineure » de tel ou tel d'entre eux contamine leur compréhension générale. Dans ce cas, loin de se compenser ou d'interagir positi- 
vement, certains registres, cadres des réponses des élèves ne leur permettent pas de profiter pleinement de la scolarité comme instance transformatrice.

\section{NOTES}

1. L'enquête Pisa évalue les compétences de jeunes de 15 ans. En France ces jeunes peuvent se trouver, selon leur niveau scolaire, en $3^{e}$ ou en $2^{\text {nde }}$. La population d'élèves de collège en retard d'un an que nous avons interrogée pour cette recherche se situe dans des collèges de ZEP de la périphérie parisienne.

2. En compréhension de l'écrit, la population d'élèves sous le niveau 2 (seuil en dessous duquel les compétences minimales ne sont pas maîtrisées) a augmenté de près de $30 \%$ en France entre 2000 et 2009, alors qu'elle baissait de $6 \%$ en moyenne dans les 26 pays de l'OCDE. Quant aux élèves français sous le niveau 1 , les plus faibles, leur proportion a presque doublé (passant de 4,2\% à 7,9\%) entre 2000 et 2009, tout comme en culture mathématique entre 2003 et 2009 (elle est passée de 5,6\% à 9,5\%). Olivier Rey (2011). «PISA: ce que l'on en sait et ce que l'on en fait». Dossier d'actualité Veille et Analyses, n 66, octobre.

3. L'équipe Escol créée en 1987 à l'université de Paris est aujourd'hui composante du Circeft, laboratoire de Paris 8 co-habilité avec l'UPEC.

4. Recherches sur la Socialisation, l'Enseignement, les Inégalités et les Différenciations dans les Apprentissages

5. Voir Rochex \& Crinon, J. (2012)

6. Notre travail sur les données PISA compréhension de l'écrit, utilise les productions des élèves moins pour l'analyse de la compréhension que pour celle de l'interprétation que les élèves font des activités qui leur sont proposées et des significations qu'ils construisent à partir des documents emblématiques du curriculum actuel.
7. Cf par exemple Spirale $N^{\circ} 47$ « La culture de l'expression $»(2011)$.

8. Dans un précédent article (Bautier $E$ al. 2006), nous avons tenté une catégorisation en termes de registres. Elle concernait à l'époque les mobilisations qui pouvaient détourner les élèves des réponses attendues aux questions de l'enquête PISA. Dans le présent article, notre utilisation de la notion de registre vise à identifier les attentes de l'école analysées à la lumière des évolutions curriculaires 9. Ces évolutions ne font qu' « aggraver » et complexifier les difficultés de certains élèves décrites par Lahire (1993) dans «Culture écrite et inégalités scolaires », Lyon, PUL. 10. Il est évident que les difficultés des élèves sont d'autant plus grandes que l'étayage, voire l'apprentissage de ces pratiques cognitives autant que discursives sont faibles au sein de la classe.

11. Ainsi, « L'évaluation PISA s'intéresse beaucoup plus aux compétences mobilisant des connaissances qu'aux connaissances elles-mêmes. Les élèves ne sont pas évalués sur des connaissances au sens strict mais sur leurs capacités à mobiliser et appliquer celles-ci dans des situations variées [...] » La lecture au collège. Le bilan des évaluations PISA, CNDP, 2011, p. 9

12. Deux articles peuvent compléter les informations que nous n'avions pas la place ici de fournir: Bautier et alii (2005), «Les performances en littéracie et l'hétérogénéité des univers mentaux mobilisés par les élèves », Cadmo, p. 43-64 et Bautier et alii (2006), « Performances en littéracie, modes de faire et univers mobilisés par les élèves: analyse secondaire de l'enquête PISA 2000 », Revue française de pédagogie, p. 85-102.

13. Certes une des réponses en QCM proposait cette solution et a peut-être contaminé les autres réponses pour ces élèves 


\section{RÉFÉRENCES}

Barrère, A. (2011). L'éducation buissonnière. Quand les adolescents se forment par eux-mêmes. Paris : Armand Colin.

Bautier, É., \& Rayou, P. (2013). Les inégalités d'apprentissage: Programmes, pratiques et malentendus scolaires. Paris : PUF 2nde édition.

Bautier, É., Crinon, J., Delarue-Breton, C., \& Marin, B. (2012). Les textes composites : des exigences de travail peu enseignées? Repères, 45, 63-79.

Bautier, É. (2010). Changements curriculaires : des exigences contradictoires qui construisent des inégalités. Entre littéracie étendue, segmentation et contextualisation des savoirs, in C. Ben Ayed (dir.), L'école démocratique, p. 83-93. Paris : Armand Colin.

Bautier, É., \& Rochex, J.-Y. (2007). Apprendre : des malentendus qui font la différence. In J. Deauvieau \& J.-P. Terrail (dir.), Les sociologues, l'école et la transmission des savoirs, p. 227-241. Paris : La Dispute.

Bautier, É., (Ed.). (2006). Apprendre l'école pour apprendre à l'école. Des risques de constructions des inégalités dès la maternelle. Lyon : Chronique sociale.

Bautier, É., Crinon, J., Rayou, P., \& Rochex, J.-Y. (2006). Performances en littéracie, modes de faire et univers mobilisés par les élèves. Analyses secondaires de l'enquête Pisa 2000. Revue française de pédagogie, 157,p. 85-101.

Bautier, É., Crinon, J., Rayou, P., \& Rochex, J.-Y. (2005). Les performances en littéracie et l'hétérogénéité des univers mentaux mobilisés par les élèves. Cadmo, roma tre, xiii, 2. p. 43-64.

Bautier, É., (2005). Mobilisation de soi, exigences langagières scolaires et processus de différenciation. Langage et société, n 111, p. 51-72.

Bautier, É., (1989). Aspects socio-cognitifs du langage. Quelques hypothèses. Langage et société, n 47, p. 5584.

Beck, U., (2001). La société du risque. Sur la voie d'une autre modernité. Paris : Aubier.

Belenguier, M.-P. (2009). Élèves en difficulté lecteurs de littérature, approches sociolinguistiques et culturelles. Mémoire de master2, sciences de l'éducation, université de Paris 8.
Bernstein, B. (1967). Open schools - open society?, New Society, 14, p. 351-353.

Bernstein, B. (1975). Langage et classes sociales. Paris : Minuit.

Bernstein, B. (2007). Pédagogie, contrôle symbolique et identité. Théorie, recherche, critique, Laval : Pul.

Bonnéry, S. (2007). Comprendre l'échec scolaire. Élèves en difficultés et dispositifs pédagogiques. Paris : La Dispute.

Bourdieu P., (1979). La distinction. Critique sociale du jugement. Paris : Minuit.

Bourdieu, P., \& Gros F. (Eds.) (1989). Principes pour une réflexion sur les contenus de l'enseignement. Paris: Imprimerie nationale.

Charlot B. (1997), Du rapport au savoir. Éléments pour une théorie. Paris: Anthropos.

Charlot, B., Bautier, É., \& Rochex, J.-Y. (1992). École et savoir dans les banlieues... et ailleurs. Paris: Armand Colin.

Chartier, A.-M. (2005). L'école entre crise des croyances et crise des pratiques. L'exemple de la lecture à voix haute. In Jacquet-Francillon, F. \& Kambouchner, D. (dir.), La crise de la culture scolaire, p. 227-261. Paris : PUF.

Chiss, J.-L. (2011).Langage, société et école : de quelques considérations sur la « culture de l'expression », Spirale, 47, p. 15-20.

Coulangeon, Ph. (2011). Les métamorphoses de la distinction. Inégalités culturelles dans la France d'aujourd'hui. Paris : Grasset.

Durkheim, É. (1990/1938). Lévolution pédagogique en France. Paris : PUF.

Rockwell, E. (2000). Tres planos para el estudio de las culturas escolares : el desarollo humano desde una perspectiva histórico-cultural . Interações (Brasil) V, 009 , p. 11-25.

Stavrou, S. (2008). La recontextualisation à l'épreuve de la sociologie empirique des curricu-la : éléments de recherche sur la régionalisation des savoirs In Frandji D., $\&$ Vitale, P. (dir.). Actualité de Basil Bernstein. Savoir, pédagogie et société, p. 171-187. Rennes : PUR.

Vygotski, L. (1997/1934). Pensée et langage. Paris : La Dispute.

Zanten, A. van (2012). L'école de la périphérie. Paris : PUF. 


\title{
Annexes
}

\section{GUIDE DE CONDUITE POUR LES ETUDIANTS PARTICIPANT AU PROGRAMME D'ECHANGES ${ }^{1}$}

\author{
Une attitude positive et le désir d'apprendre de nouvelles choses constituent le point de départ d'une année \\ réussie. \\ La différence est un atout : soyez vous-même ! \\ Respectez la culture locale et acceptez qu'elle soit différente de la vôtre : soyez tolérants ! \\ Faites circuler des informations sur votre pays d'origine : emportez des brochures, des photographies et \\ toute autre documentation utile. N'oubliez pas d'en inclure sur votre région d'origine. \\ Investissez-vous dans toutes les activités de loisirs intéressantes. L'école vous en proposera un large \\ éventail. \\ Les contrariétés font également partie du programme durant l'année d'échanges. Ne vous laissez pas abattre, \\ transformez-les plutôt en expériences positives : toute expérience est une occasion d'apprendre ! \\ Que cette année se révèle une réussite dépend en grande partie de vous-même : agissez en conséquence ! \\ N'oubliez pas de sourire, même les jours où il fait gris ! \\ Lorsque vous êtes confrontés à des problèmes, n'hésitez pas à demander conseil. Vous n'êtes pas seuls ! \\ Ne craignez pas de faire des fautes : dès le premier jour utilisez sans hésiter la langue du pays d'accueil, et \\ vous verrez que les gens vous comprendront. \\ Vous comprendrez vite l'importance des mots "s'il vous plaît" et "merci". Assurez-vous qu'ils font bien \\ partie de votre vocabulaire ! \\ Profitez de cette année. Faites-en une des meilleures que vous ayez jamais vécue. \\ À la fin de l'année, dites-vous ceci : "ce n'est pas terminé - cela ne fait que commencer !" \\ Une fois rentré chez vous, n'oubliez pas les amis avec lesquels vous avez lié connaissance pendant l'année \\ passée à l'étranger. Restez en contact !
}

Référez-vous au " Guide de conduite pour les étudiants participants au programme d'échanges " qui figure à la page ci-contre pour répondre aux questions qui suivent.

Question 18: GUIDE DE CONDUITE

Ce Guide insiste surtout sur l'importance :

A d'être un bon ambassadeur de votre pays.

B d'établir de bonnes relations avec votre famille d'accueil.

$\mathrm{C}$ de vous faire autant de nouveaux amis que possible dans le pays d'accueil.

$\mathrm{D}$ de faire de votre programmes d'échange une expérience enrichissante pour toutes les personnes concernées.

Question 19: GUIDE DE CONDUITE

Quelle est, parmi les phrases suivantes, celle dont le sens est le plus proche de celui du point 2 du Guide?

A Faites un effort pour vous adapter à la culture de ceux qui vous entourent.

B Vous serez plus intéressant si vous n'êtes pas comme tout le monde.

C Ne vous préoccupez pas de la culture du pays d'accueil.

D Vous ne pouvez pas être comme ceux qui vous entourent, même si vous essayez.

Question 20 : GUIDE DE CONDUITE

Lisez le point 13 du Guide

Que veut-on dire par "cela ne fait que commencer" ?

A C'est le début d'une nouvelle année scolaire.

B D'autres étudiants commencent leur année de programmes d'échanges.

C Vous êtes sur le point d'entrer dans un environnement inconnu.

D La vie chez vous sera différente après une année de programme d'échanges.

1 Un étudiant participant à un programme d'échange quitte son foyer pour poursuivre ses études à l'étranger pendant un certain temps, généralement une année, au cours de laquelle il vit dans une famille d'accueil. 


\section{MACONDO}

Éblouis par tant d'inventions, et si merveilleuses, les gens de Macondo ne savaient par où commencer à s'étonner. Ils passaient des nuits blanches à contempler les pâles ampoules alimentées par le groupe électrogène qu'avait rapporté Aureliano le Triste lors du second voyage du train, et à l'obsédant teuf-teuf duquel on ne s'habitua qu'à la longue, péniblement. Ils furent indignés par les vivantes images que le riche commerçant Don Bruno Crespi projetait dans le théâtre aux guichets en gueules de lion, à cause d'un personnage mort et enterré dans certain film, sur le malheur duquel on versa des armes amères, et qui reparut bien vivant et transformé en arabe dans le film suivant. Le public, qui payait deux centavos pour partager les mésaventures des personnages, ne put supporter cette inqualifiable moquerie et brisa tous les sièges. Le maire, cédant aux instances de Don Bruno Crespi, dut faire une proclamation publique pour expliquer que le cinéma n'était qu'une machine à illusions, laquelle ne méritait pas ces débordements passionnels du public. À la suite de cette décevante explication, beaucoup estimèrent qu'ils avaient été victimes d'une nouvelle et spectaculaire affaire de gitans, si bien qu'ils choisirent de ne plus se rendre au cinéma, considérant qu'ils avaient assez de leurs propres peines pour aller encore pleurer sur les malheurs d'êtres imaginaires.

Le texte de la page est extrait d'un roman. Dans cette partie du récit, on vient d'introduire le chemin de fer et l'électricité dans la petite ville imaginaire de Macondo, et d'ouvrir le premier cinéma.

Référez-vous à ce texte pour répondre aux questions suivantes.

\section{Question 1 :}

Dans les films, quel était l'élément qui a mis en colère les gens de Macondo ?

\section{Question 2 :}

À la fin du passage, pourquoi les gens de Macondo ont-ils choisi de ne plus se rendre au cinéma?

A Ils voulaient s'amuser et se distraire, mais ils ont trouvé que les films étaient réalistes et déprimants.

B Ils n'avaient pas les moyens de se payer le prix du billet.

C Ils préféraient garder leurs émotions pour des occasions de la vie réelle.

D Ils cherchaient à s'impliquer émotionnellement, mais ils ont trouvé les films ennuyeux, peu convaincants et de mauvaise qualité.

\section{Question 3 :}

Qui sont les "êtres imaginaires" dont on parle à la dernière ligne du texte ?

A Des fantômes

B Des inventions de champ de foire

C Des personnages de films

D Des acteurs

\section{Question 4 :}

Êtes-vous d'accord avec le jugement final des gens de Macondo à propos de l'intérêt du cinéma ? Expliquez votre réponse en comparant votre attitude et la leur à l'égard des films 